\title{
Use of a helical composite free flap for alar defect reconstruction with a supermicrosurgical technique
}

\author{
Hyung Hwa Jeong, Dong Hoon Choi, Joon Pio Hong, Hyun Suk Suh \\ Department of Plastic and Reconstructive Surgery, Asan Medical Center, University of Ulsan College of Medicine, Seoul, Korea
}

The highly contoured nature of the nose and the abundant free margin makes it especially difficult to reconstruct. In this report, we describe the use of a new helical rim free flap technique for the reconstruction of full-thickness nasal alar defects via supermicrosurgery. Briefly, after a wide excision with a margin of $0.7 \mathrm{~cm}$, an alar defect with a size of $1 \times 1 \times 0.5$ $\mathrm{cm}$ was obtained, which included the full thickness of the skin, mucosa, and lower lateral cartilage. Vessel dissection was performed in a straightforward manner, starting from the incision margin for flap harvest, without any further dissection for reach the greater trunk of the superficial temporal artery. The flap was inset in order to match the contour of the contralateral ala. We closed the donor site via rotation and advancement. No donor site morbidity was observed, despite the presence of a small scar that could easily be covered with hair. The alar contour was satisfactory, and the patient was satisfied with the results. The supermicrosurgical technique did not require further dissection to identify the vessels for anastomosis, leading to better cosmetic outcomes and a reduced operating time.

Keywords Nose / Free tissue flaps / Carcinoma, squamous cell
Correspondence: Dong Hoon Choi Department of Plastic and Reconstructive Surgery, Asan Medical Center, University of Ulsan College of Medicine, 88 Olympic-ro 43-gil, Songpa-gu, Seoul 05505, Korea

Tel: $+82-2-3010-3600$

Fax: +82-2-476-7471

E-mail: donghwn@naver.com

\section{INTRODUCTION}

The introduction of the subunit principle changed the manner in which surgeons manage nasal defects [1]. The reconstruction of nasal defects in areas such as the alae and columella remains challenging due to the complex 3-dimensional nature of these structures. Moreover, the highly-contoured nature of the nose and the abundant free margin make such reconstruction even more difficult.

Many different techniques have been described for full-thickness nose reconstruction. Pedicled flaps, such as the forehead flap and nasolabial flap, are frequently used, although they pres- ent limitations in terms of accurately imitating the contour of the nasal rim. An unfavorable donor site scar is another limitation of pedicled flaps that is difficult to resolve. Small defects, $<2 \mathrm{~cm}$ in diameter, can be reconstructed using a composite helical graft [2]. However, in cases with defects $>2.0 \mathrm{~cm}$, a composite graft may not provide stable viability. Hence, free tissue transfer is considered necessary for larger full-thickness alar defects. The use of a free composite auricular flap based on the superficial temporal artery was first described in 1984 [3]. A variation of this flap, including the reverse-flow design of the free helical rim flap, was subsequently described by Lin et al. [3] .

However, pedicle shortage remains a major problem with free 
auricular composite tissue transfer [3]. Several surgeons have attempted to overcome this limitation [1]. In this report, we describe the use of a new helical rim free flap technique for the reconstruction of full-thickness nasal alar defects via supermicrosurgery. Supermicrosurgery involves the microanastomosis of vessels with calibers $<0.8 \mathrm{~mm}$. Although a composite graft can be a choice for small defects, as seen with zone 1 finger replantation, when microsurgical repair is possible, it may have further benefits and lead to improved outcomes [4].

The superficial temporal artery runs through the preauricular area and gives off at least two branches to the ear: the anterior auricular artery and superior auricular artery. The superior auricular artery runs anterior to the auricular helix, passes superior to the auricle border, and anastomoses with the retroauricular artery, which itself arises from the external carotid artery [5-7]. A previous cadaveric anatomical study performed by Zhang et al. [8] (with a total of six ears) confirmed that the branches of the superficial temporal artery supply blood to the upper twothirds of the helical rim and the hairless preauricular area. Moreover, that study confirmed that the superficial temporal vein does not have any valves.

\section{CASE}

A 43-year-old woman without any specific previous medical or surgical history presented to Asan Medical Center with a chief complaint of a skin tumor on her right nasal ala. A punch biopsy conducted at another institution indicated the presence of a squamous carcinoma with positive margins. On the metastasis work-up, no evidence of distant metastasis was detected. Singlestage wide excision and reconstruction was scheduled, with mi-

\section{Fig. 1. CT angiography findings of recipient vessel}

The superficial temporal artery and its branch to the helical rim were found in a computed tomography (CT) angiography fusion image.

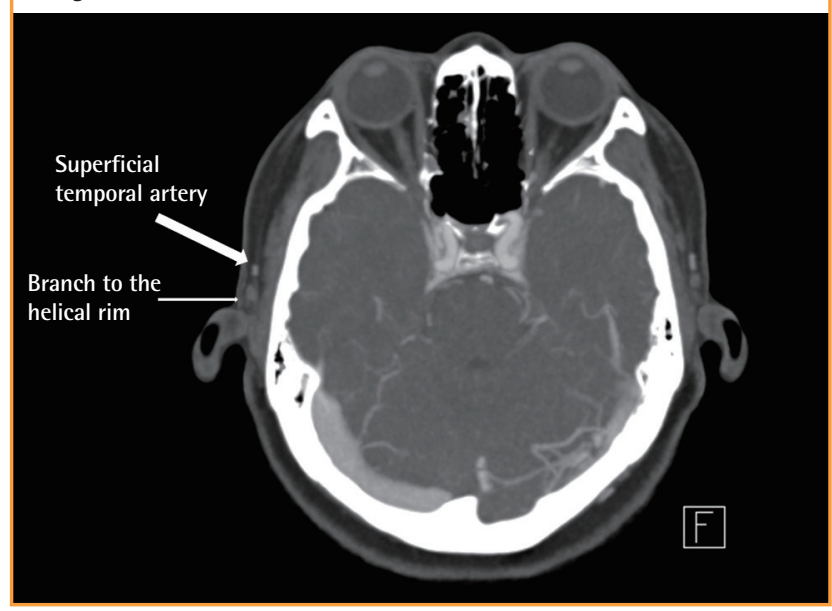

crosurgical reconstruction of the right ala using a helical free flap. Prior to surgery, the vessels adjacent to the defect and donor sites were assessed via computed tomography (CT) angiography. The superficial temporal artery and its branch to the upper portion of the helical rim could be identified on axial CT angiography (Fig. 1). Hand-held Doppler tracing also indicated the superficial temporal arterial branch to the helix and angular artery trunk, as well as its branch to the alae.

We determined the excision margin according to the methods previously described by Thomas et al. [9]. Briefly, after wide excision with a margin of $0.7 \mathrm{~cm}$, an alar defect with a size of $1 \times 1 \times 0.5 \mathrm{~cm}$ was obtained, which included the full thickness of the skin, mucosa, and lower lateral cartilage (Fig. 2). Moreover, approximately $80 \%$ of the ala was missing. We designed an ipsilateral ear helical free flap nearly the same size of the defect, hoping there would be minimal contracture after microanastomosis. Vessel dissection was performed in a straightforward manner, starting from the incision margin for flap harvest, without any further dissection of reach the greater trunk of the superficial temporal artery (Fig. 3). The harvested flap had a vascular pedicle of an anonymous branch, running from the branch to the helix of the superficial temporal artery. Pedicle dissection was performed under microscopic magnification and $\mathrm{a} \times 4$ loupe.

For recipient vessel dissection, we dissected a branch of the angular artery through the excision defect, without the need for any additional incision to identify the trunk. An anonymous branch of the angular artery was dissected. The diameters of both the recipient and donor vessel were $<0.8 \mathrm{~mm}$, consistent with our inclusion criteria related to supermicrosurgery.

Microanastomosis was conducted for one artery and one vein. The perforator of the superficial temporal artery was anasto-

\section{Fig. 2. Image of the defect after resection}

After wide excision with $0.7 \mathrm{~cm}$ of extra margin, a full-thickness defect at the right ala with a size of $1 \times 1 \times 0.5 \mathrm{~cm}$ was obtained.

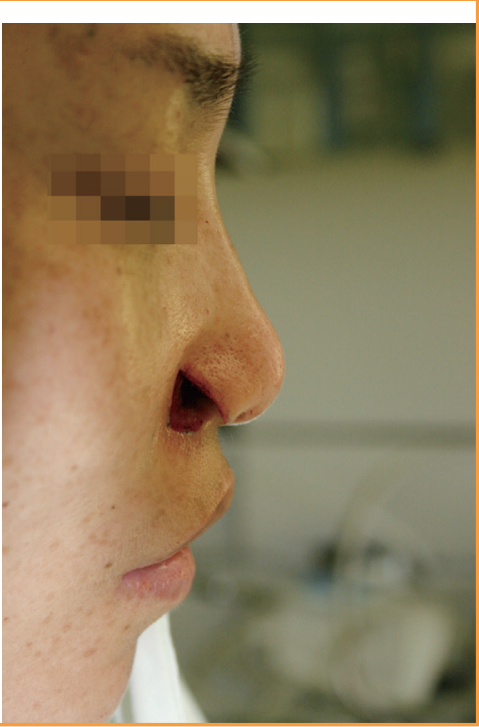




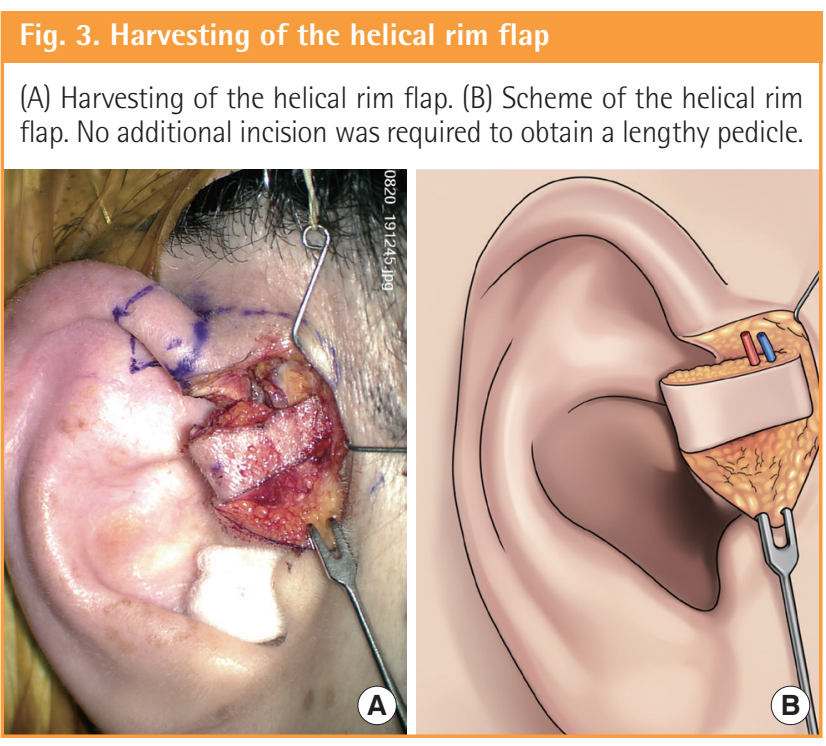

Fig. 4. Defect reconstruction with helical rim flap

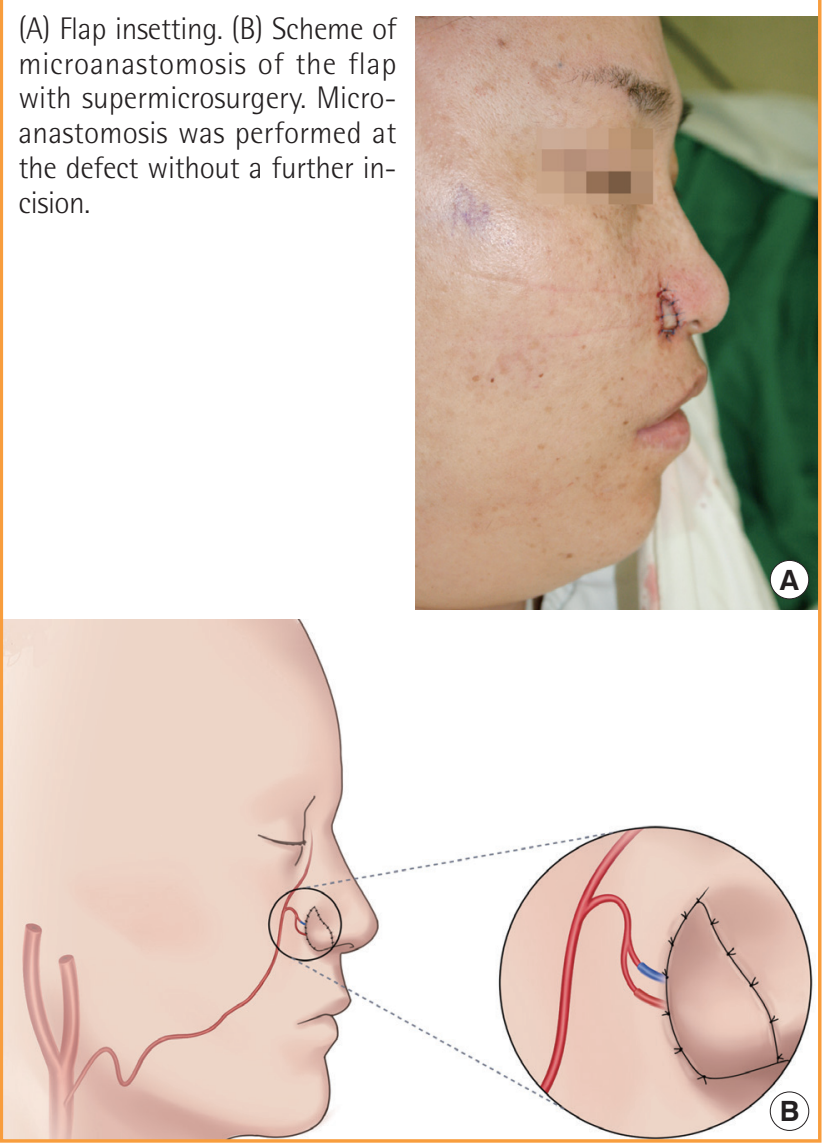

mosed to the branch of the angular artery in an end-to-end fashion. Moreover, vein anastomosis was performed between both the venae comitantes in an end-to-end fashion (Fig. 4B). The flap was inset in order to match the contour of the contralateral ala. We closed the donor site via rotation and advancement. Fig. 4A shows the immediate postoperative findings after insetting.

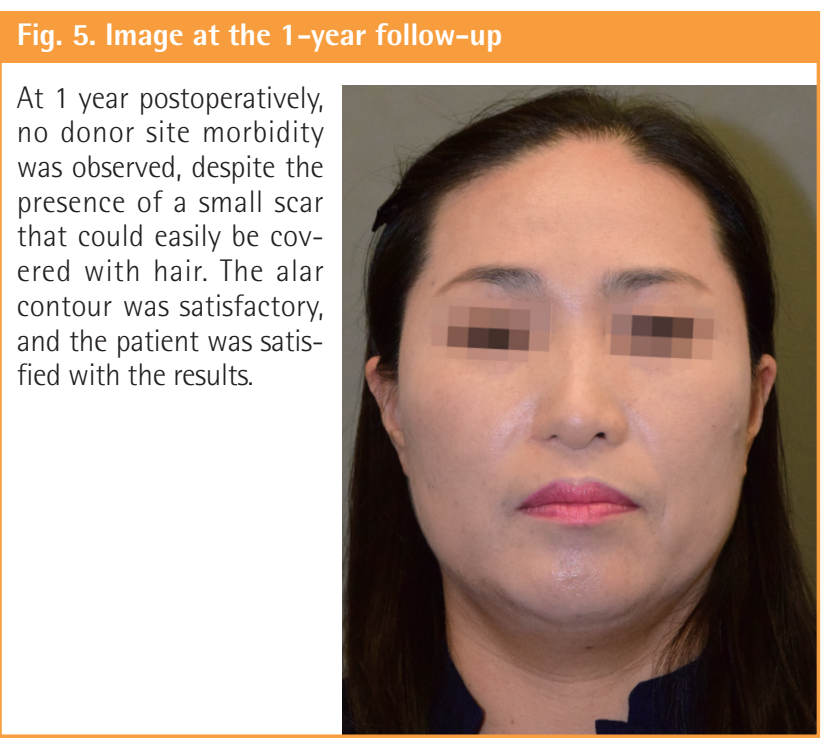

The flap was monitored with inspection of the color and contour. Doppler imaging was also used to assess the state of the pedicle. In the present case, the flap survived without any venous congestion or necrosis. Moreover, the donor site did not exhibit necrosis or deformity. At 6 months and 1 year (Fig. 5) postoperatively, no donor site morbidity was observed, despite the presence of a small scar that could easily be covered with hair. The alar contour was satisfactory, and the patient was satisfied with the results. However, the caliber of the nostril was reduced compared to the preoperative dimensions.

\section{DISCUSSION}

Commonly used pedicled flaps include the forehead flap, nasolabial flap, and postauricular flap. These flaps are inherently skin flaps, and hence cannot provide adequate structural support or restore a thin and highly-contoured surface. The strength of the free helical flap, as compared to these other flaps, is that it enables the single-stage reconstruction of nasal defects, without the need for multiple operations to delay or divide the flap. Moreover, for the staged reconstruction of full-thickness alar defects with local flaps, delayed primary cartilage grafting or additional skin grafting is required to expand the natural lining or obtain structural stability. Moreover, to restore the nasal lining, folding of the skin paddle of the flap is required, which can compromise the circulation of the skin flap [10]. Alternatively, skin grafting can be used. These problems can be resolved by using an ascending helix, due to its composite structure. Furthermore, full-thickness nasal defects are reportedly associated with major complications in cases of nasal reconstruction with a forehead flap [11]. Full-thickness defects invariably require structural grafting, which lacks an intrinsic blood supply. 
A composite chondrocutaneous graft is comparable to a helical free flap. Its skin texture and layer offer an excellent tissue match [8]. However, the lack of stable vascularity limits the use of free grafts for small alar defects. The transfer of vascularized tissue provides a long-lasting option for larger defects [12]. Moreover, the potential for monitoring the flap during admission circumvents the limitations of grafting. In particular, the prick test is a reliable method for assessing the condition of the flap. By using the helical free flap, we tried to overcome these unpredictable issues that occur in the composite graft approach. A helical free flap provides an intimate contour and structure to the recipient site. It is almost impossible to reconstruct a thin and precise alar margin using conventional procedures. The reconstruction of nasal defects does not simply involve "filling a hole" [8], given the complex structure and presence of several subunits. Hence, the restoration of the subunits should form the basis of the reconstruction [8]. In our case, there was significant contracture during follow-up, without apparent incidents after reconstruction. We may suspect the flap may have undergone contracture for various reasons, including a lack of structural support on the base of the flap. Nevertheless, this approach may be an option for future reconstruction of larger defects in this difficult region.

A donor site scar can be a source of permanent stigma for those who have undergone nasal defect reconstruction with a local flap. The use of a free flap can avoid unfavorable donor site scars in the face. However, the skin and fat composition of free flaps cannot mimic the 3-layer composition of the nasal alae.

Furthermore, by using the supermicrosurgical technique, the advantages of the helical free flap can be maximized. The shortness of the vascular pedicle is an inherent pitfall of free helical flap use. To overcome this, a reverse-flow design, an additional incision for obtaining a longer recipient vessel, and vascular grafts have previously been used $[1,8]$. However, in the present case, we performed the reconstruction using the branch and perforator, rather than the main trunk, at both the donor and recipient sites. Zhang et al. [8] indicated that the facial or superficial temporal vessels were the best options for the recipient vessel. The supermicrosurgical technique does not require further dissection to identify the vessels for anastomosis, leading to better cosmetic outcomes and reduced operative time.

This procedure has certain limitations that are inherent to the use of conventional helical free flaps. Microsurgical skill is essential for achieving good results. Moreover, the shrinkage of the transferred flap makes it difficult to evaluate the outcomes in the long term. The use of a nasal retainer for preventing the collapse of the structure might prevent flap shrinkage and aid in restoring the nasal caliber.

\section{NOTES}

\section{Conflict of interest}

No potential conflict of interest relevant to this article was reported.

\section{Patient consent}

The patient provided written informed consent for the publication and the use of her images.

\section{REFERENCES}

1. Castello JR, Taglialatela Scafati S, Sanchez O. Bilateral nasal ala reconstruction of the cocaine-injured nose with 2 free reverse-flow helical rim flaps. Ann Plast Surg 2014;73:304-6.

2. Driscoll BP, Baker SR. Reconstruction of nasal alar defects. Arch Facial Plast Surg 2001;3:91-9.

3. Lin SD, Lin GT, Lai CS, et al. Nasal alar reconstruction with free "accessory auricle". Plast Reconstr Surg 1984;73:827-9.

4. Kim KS, Eo SR, Kim DY, et al. A new strategy of fingertip reattachment: sequential use of microsurgical technique and pocketing of composite graft. Plast Reconstr Surg 2001;107:73-9.

5. Cordova A, Pirrello R, D’Arpa S, et al. Superior pedicle retroauricular island flap for ear and temporal region reconstruction: anatomic investigation and 52 cases series. Ann Plast Surg 2008;60:652-7.

6. Cordova A, D’Arpa S, Pirrello R, et al. Retroauricular skin: a flaps bank for ear reconstruction. J Plast Reconstr Aesthet Surg 2008;61(Suppl 1):S44-51.

7. Song R, Song Y, Qi K, et al. The superior auricular artery and retroauricular arterial island flaps. Plast Reconstr Surg 1996;98:657-67.

8. Zhang YX, Yang J, Wang D, et al. Extended applications of vascularized preauricular and helical rim flaps in reconstruction of nasal defects. Plast Reconstr Surg 2008;121:1589-97.

9. Thomas DJ, King AR, Peat BG. Excision margins for nonmelanotic skin cancer. Plast Reconstr Surg 2003;112:57-63.

10. Menick FJ. A 10-year experience in nasal reconstruction with the three-stage forehead flap. Plast Reconstr Surg 2002; 109:1839-55.

11. Little SC, Hughley BB, Park SS. Complications with forehead flaps in nasal reconstruction. Laryngoscope 2009;119:1093-9.

12. Holzmann D, Forster NA, Vital D, et al. Reconstruction of defects involving the nasal ala and the nasolabial fold: the role of the microvascular prehelical rim graft. ORL J Otorhinolaryngol Relat Spec 2015;77:255-61. 